\title{
Development of HID-Based Motion Recognition Device
}

\author{
Won-Hyuck Choi ${ }^{1}$, Da-Un Kim ${ }^{2}$ and Min-Seok Jie ${ }^{3}$ \\ 1, 2, ${ }^{3}$ Department of Avionics Engineering Hanseo University \\ ${ }^{1}$ choiwh@hanseo.ac.kr, ${ }^{2} k d u 0011 @$ naver.com, ${ }^{3}$ jiems@hanseo.ac.kr
}

\begin{abstract}
In this paper, we developed a three-dimensional motion recognition device based on a wireless transceiver for the HMD (Head Mounted Display) (Camera Gimbal). This device using a gyro sensor and acceleration sensor. Recognizes the three-dimensional motion of the user's head and controls the object wirelessly. Also, attached directly to the user in a HID (human interface devices) system. It utilizes the movement of the user obtained from the device. Kalman filter was used to improve the accuracy of the data. This device is linked with unmanned aircraft, to the user can deliver more accurate information and freedom of space. It can be applied in all areas for the remote control.
\end{abstract}

Keywords: Motion Sensor, Motion Recognition, Remote Control, HID, HMD

\section{Introduction}

Topic in modern technical field can be called fusion. Fusion in the modern meaning, utilizing existing one, providing new goods and services, says business activities that create added value. In particular, If ICT(Information and Communications Technologies) technologies are fused to the existing industry or other industries, Communications, consumer, such as a computer device are each other have a similar function. Because it is possible to produces of products with a high value-added, according to the characteristics of the industry [1].

Long before attempts were made fusion based on the ICT technologies in the aviation sector that require state-of-the-art technology. It is applied to the aircraft and its associated equipment has been to provide high reliability of the pilot convenience and safety, the aircraft operation and safety. Recently, the most actively areas of aviation ICT technology are fused is avionics. The characteristics of the industry of Avionics sector, it is possible to create high value-added. Also, has the advantage of high utilization of the product. Aviation ICT fusion technology, in the avionics, up to the vehicle and mobile platforms, Spin-Off is actively in progress. HMD and Augmented Reality technology that is representative of hardware and software [2].

In this paper, we developed a motion recognition device for HMD camera gimbal control of drone. An important part of the motion recognition device is s data. This is because the camera gimbal must move precisely with the movement of the user's head. The Kalman filter algorithm is used for data stabilization. So, through the motion recognition check the user's midair motion, and designing the HID-based wireless communication protocol, it is possible to control and easy remote work with the smart device.

\section{Related Studies}

\subsection{Motion Recognition}

First it is necessary a gyro sensor and an acceleration sensor (Motion Sensor) to sense the user's motion. To identify elaborate motion of user, using the gyro sensor that is not affected by the Earth's gravity as the main measurement device. The gyro sensor is a 
device that can measure the acceleration of the object is moving. Gyro sensor, it is possible to accurately measure the motion coordinate value of the user, it is possible to finely recognize the midair motion. However, a gyro sensor, gravitational acceleration and motion acceleration in the not be measured is measured using an acceleration sensor [3-4].

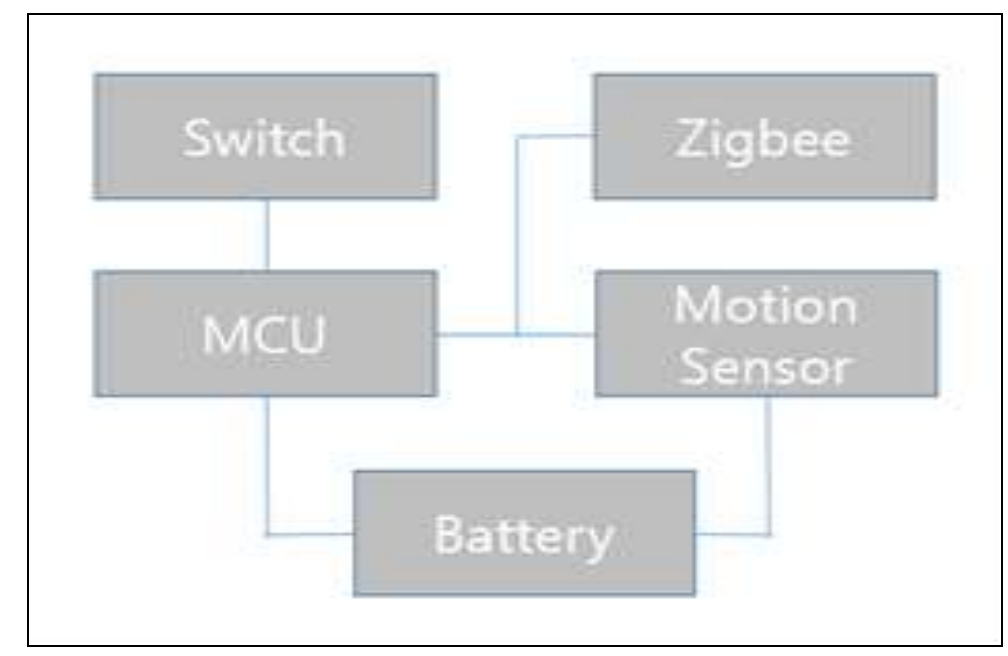

Figure 1. Block Diagram of Motion Recognition Device

\subsubsection{Gyro Sensor}

A gyro sensor is a sensor that measures the input angular velocity in given environment. The gyro sensor has design parameters such as rotation axis, moment of inertia, elasticity coefficient, load factor, and output shaft inertia. These are determined by the environment in which the gyro is used, thereby determining the stability traction and performance of the gyro. Gyro sensors can sense rotational motion and changes in orientation and therefore augment motion. Vibration gyro sensors can sense angular velocity due to the Coriolis force which is applied to a vibrating element. This motion produces a potential difference from which angular velocity is sensed. The angular velocity is converted into an electrical signal output.

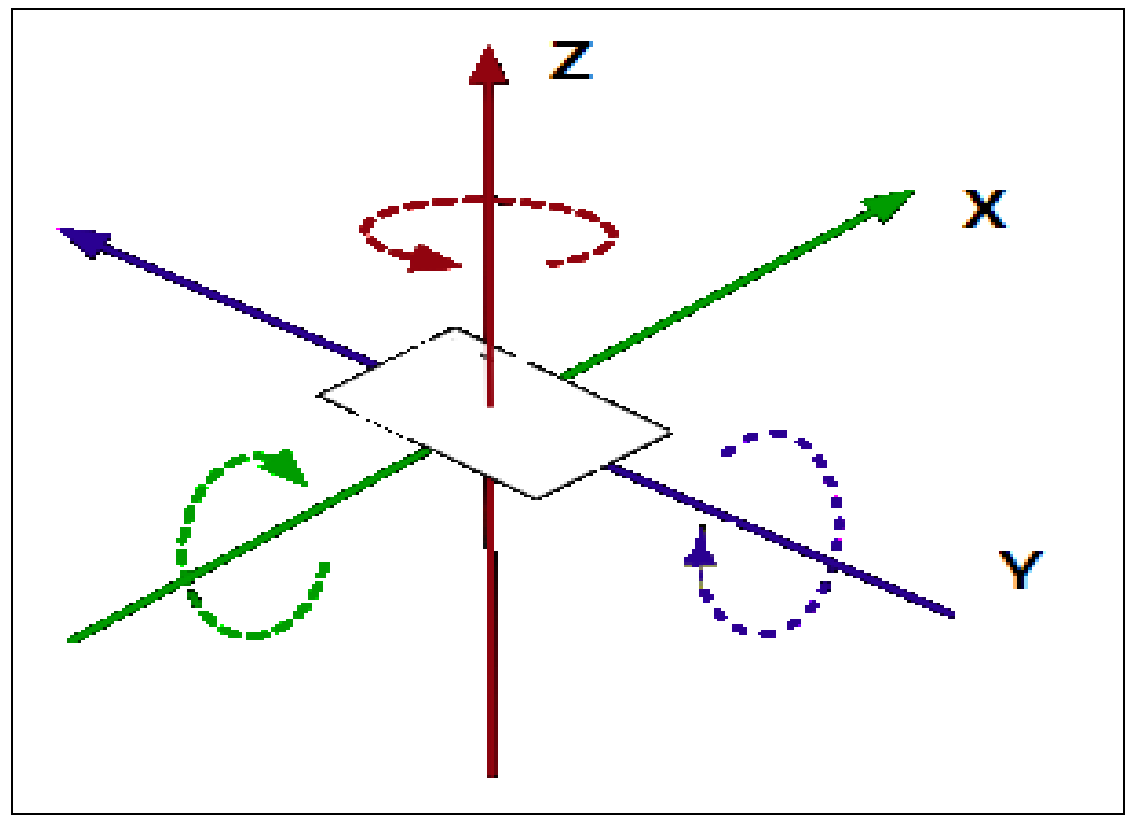

Figure 2. Gyro Sensor 
Coriolis force equation.

$$
F=m r(w+\Omega)^{2}=m r w^{2}+2 m r \Omega
$$

In this equation, $v=r w$ and $m$ is the rotational speed with respect to the plate. The first term of Equation (1) is the centrifugal force measured at the coordinates based on the flat plate. The second term is the force of the vector product of the rotation $\mathrm{w}$ of $\mathrm{m}$ and the rotation $\Omega$ of the plate. It is a force perpendicular to $\mathrm{v}$ and $\Omega$, and this is the force of Coriolis.

\subsubsection{Accelerometer Sensor}

The acceleration sensor outputs the magnitude of the acceleration applied to the object, and the type is divided by the number of axis such as 1,2 , and 3axis. In the case of a three-axis acceleration sensor, the acceleration can be measured in three-dimensional space in the $\mathrm{x}, \mathrm{y}$, and $\mathrm{z}$ axis directions. Basically, since the acceleration sensor detects gravitational acceleration in a state where it is still stopped, it outputs a value of -g in the $\mathrm{z}$-axis direction.

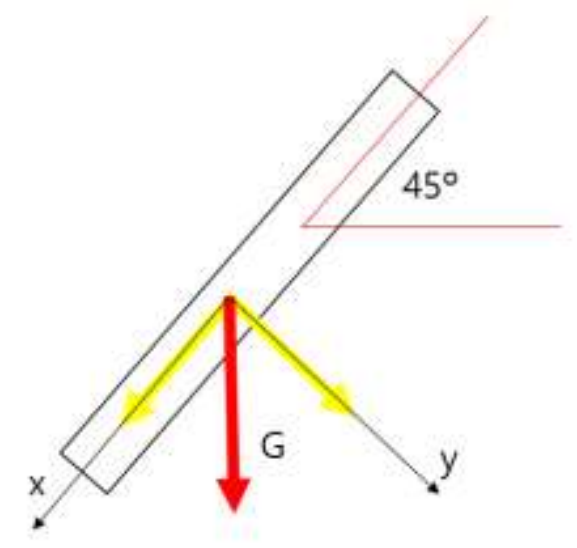

Figure 3. Accelerometer Sensor

\subsection{HID-Based Wireless Communication}

HID is a means to charge the user interface of the computer peripheral as a human interface devices. That is, almost receiving an input from a human, sometimes comprising several output functions. Developed equipment is, as a contact-type gesture-based user interface, attaching a sensor or device to the body part of the user. Utilizing the user's motion information obtained from the connected sensors and devices. Advantages of the contact method can obtain an accurate information the user gesture, it is effective in the interaction with the three-dimensional virtual object, because it can take advantage of the three-dimensional information of user motion [5].

ZigBee is a high-level communications protocol that uses a small, low-power digital radio based on IEEE 802.15.4-2003. IEEE 802.15.4-2003 is the standard for short-range personal radio networks such as lamps, electronic meters, and consumer electronics using short-range radio frequencies. ZigBee is primarily used in RF applications that require low data rates, low battery consumption, and network security. ZigBee is currently used in embedded sensors, industrial control, medical data collection, building automation, fire and theft, and home automation. ZigBee is simpler than many other protocol stacks, and the size of the ZigBee stack code is smaller than other protocols [6-7]. 


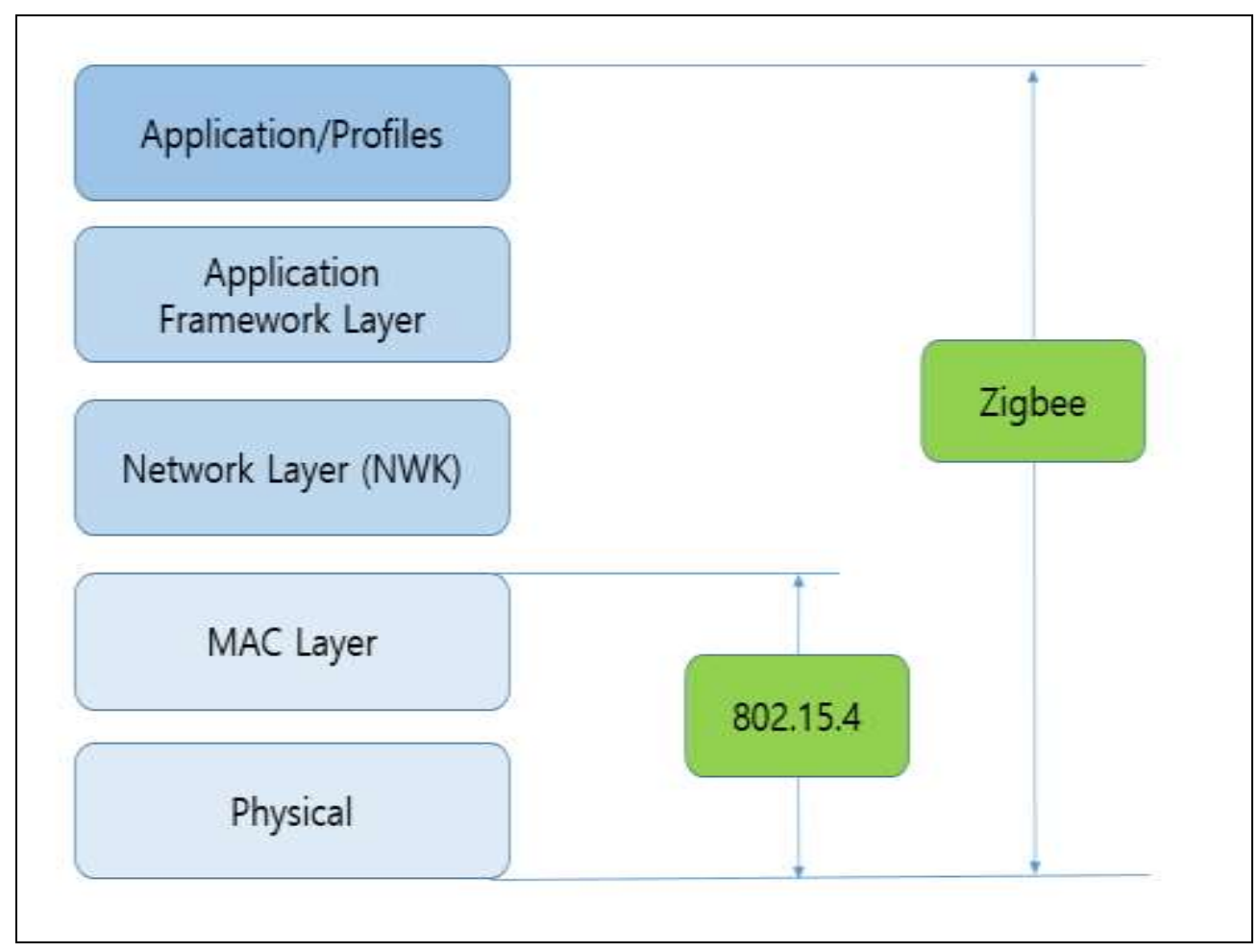

Figure 4. Zigbee Stack

ZigBee is simpler than many other protocol stacks, and the size of the ZigBee stack code is smaller than other protocols. The MAC and PHY are defined by the IEEE 802.15.4 standard. The network and application layers are defined by the actual application and Zigbee Alliance provided by the equipment designer.

\subsection{Gimbal System}

In the gimbal system, two DC motors are directly connected to the roll and pitch driving shaft, and the IMU sensor is mounted on each side to detect the attitude information of the user. An optical incremental encoder was used for feedback to each drive shaft, and the main controller used Atmega2560 [8].

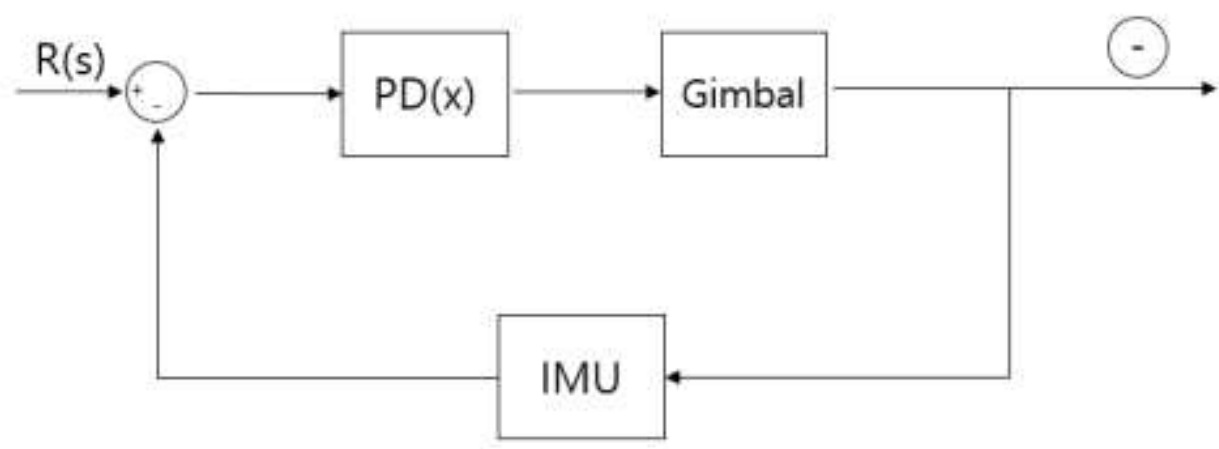

Figure 5. Control Block Diagram of Gimbal System 


\section{System Composition}

The system proposed in this paper, a gyro sensor and acceleration sensor, that is, a system that controls the objects remotely using a motion sensor. Configuration of the system is shown in Figure 1. The system is divided into the following four parts: by measuring the motion, the Command Unit that transmits the command, a Wireless Communication Unit for transferring data, Data Processing Unit for the filtering by using a Kalman filter algorithm, Control Unit for driving the objects directly. Command Unit is in the head of the user and calculates an angular value of the motion sensor. Control Unit controls the gimbals using the sensor value received from the Command Unit.

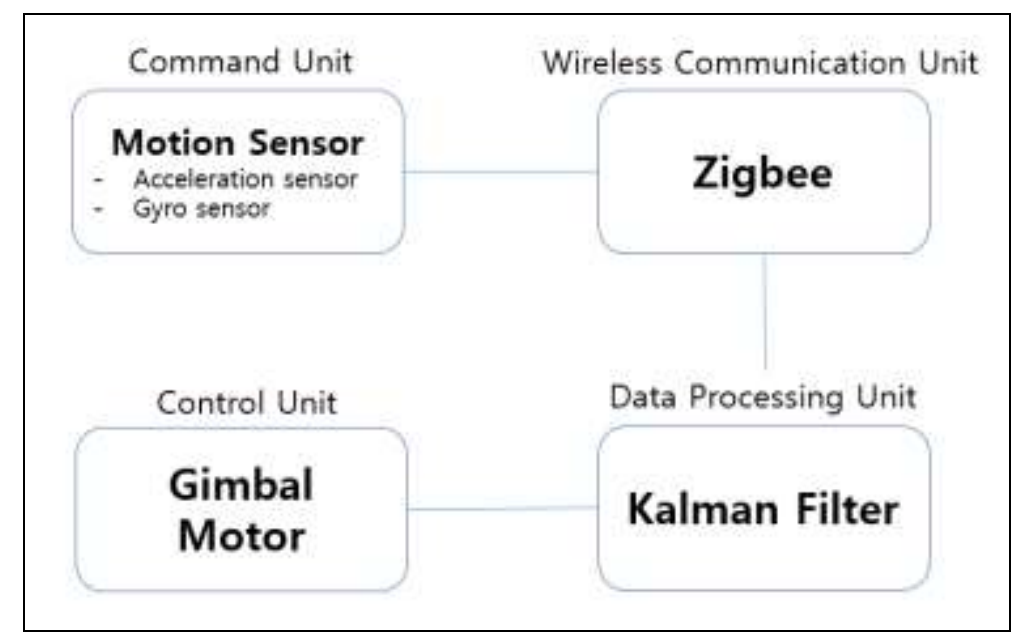

Figure 6. System Diagram

To control the gimbals from the user's head movement and remotely, the system is driven in the order shown in Figure The transmitter is located at the head of the user and calculates the angle value with the motion sensor, and the receiver controls the gimbals with the sensor value from the transmitter. A small board was made directly for the experiment. Zigbee communication module makes wireless communication between transmitter and receiver, SPI communication between board and Zigbee, and I2C communication between board and motion sensor.

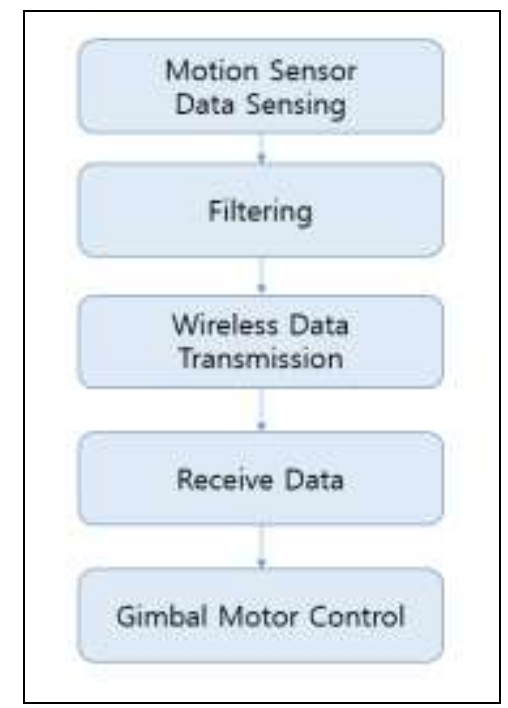

Figure 7. System Flowchart 


\subsection{Compact H/W Design}

HMD refers to a display worn on the head. Helmet, and wearable device, the $\mathrm{H} / \mathrm{W}$ size is made very compact. By eliminating unnecessary elements in $\mathrm{H} / \mathrm{W}$ as much as possible, we are made to minimize the weight so as not to inconvenience to users. The following is the $\mathrm{H} / \mathrm{W}$ circuit diagram, which is divided into two parts: one for measuring data and the other for controlling all necessary data for transmission. The data processing and transmission is a System On Chip (SoC) type, and data is transmitted through one chip and data is transferred to ZigBee. In addition, the part related to the power supply of the board includes an RGB LED portion that can display the board status and the remaining battery status.

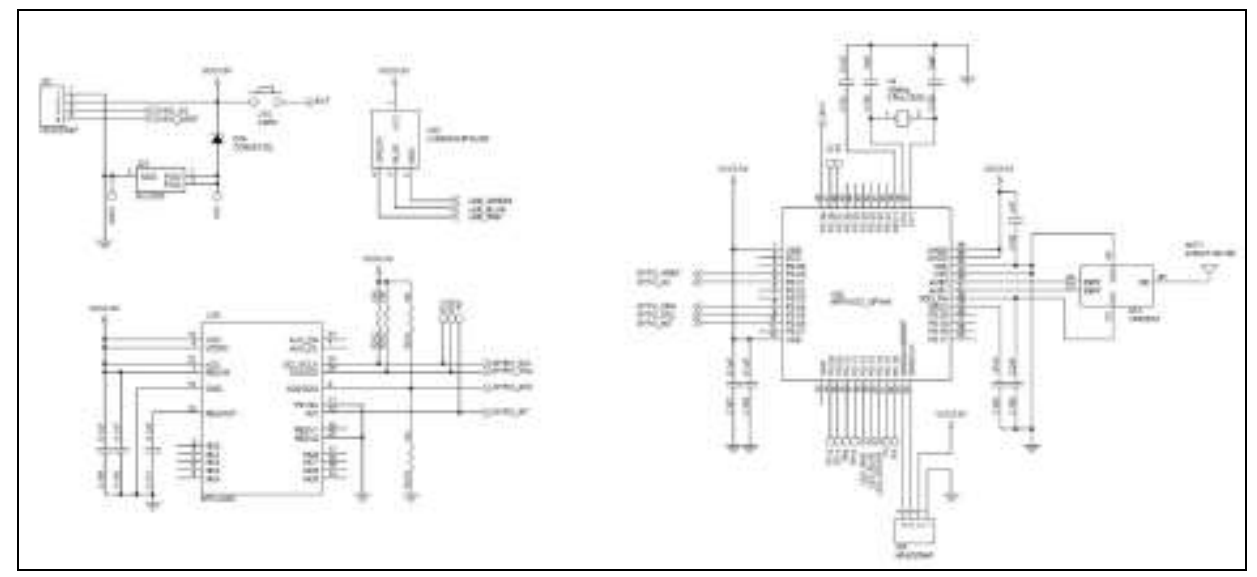

Figure 8. Circuit

\subsection{Sample Board}

The following is a sample board. The sample board injects the PCB substrate based on the design of the above $\mathrm{H} / \mathrm{W}$ and consists of IMU, communication part, LED part, and power part. The configuration includes the processor and the MPU-9250, a 9-axis sensor for calculating acceleration and angular velocity. Since the power source is mounted on the HMD and the helmet, it is supplied using a coin battery considering the size of the battery to improve portability. The size of the module was taken into consideration for the arrangement of the elements and the place where the helmet was mounted, and the size of the sample board was set to $4 \times 4 \mathrm{~cm}$ [9].

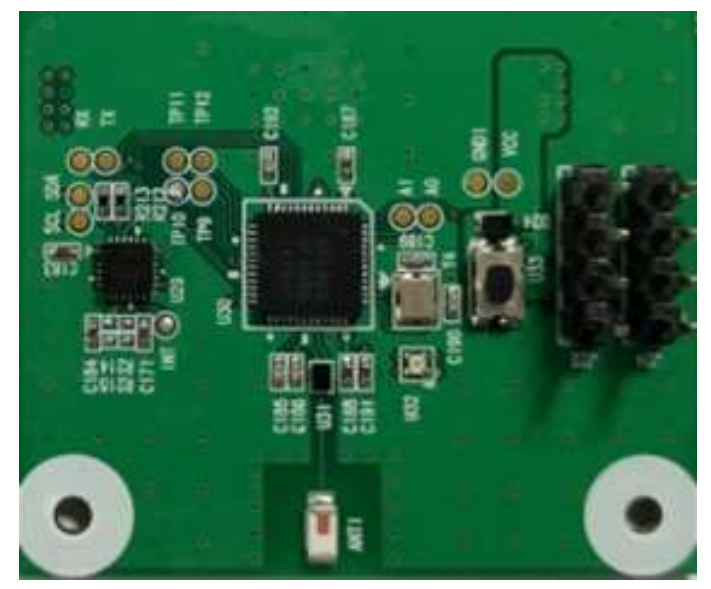

Figure 9. Sample Board 


\subsection{Simulation}

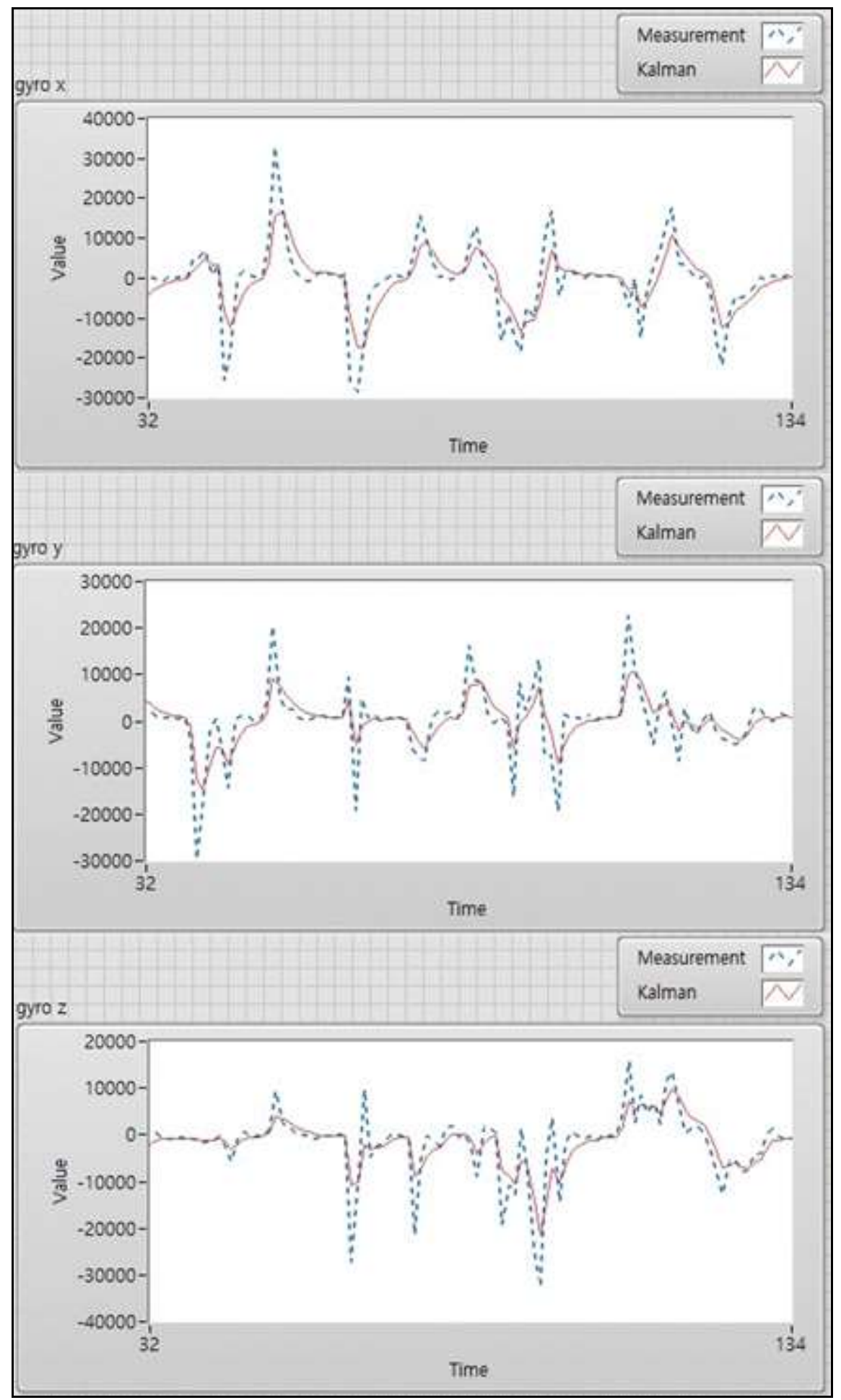

Figure 10. Gyro Sensor Graph 


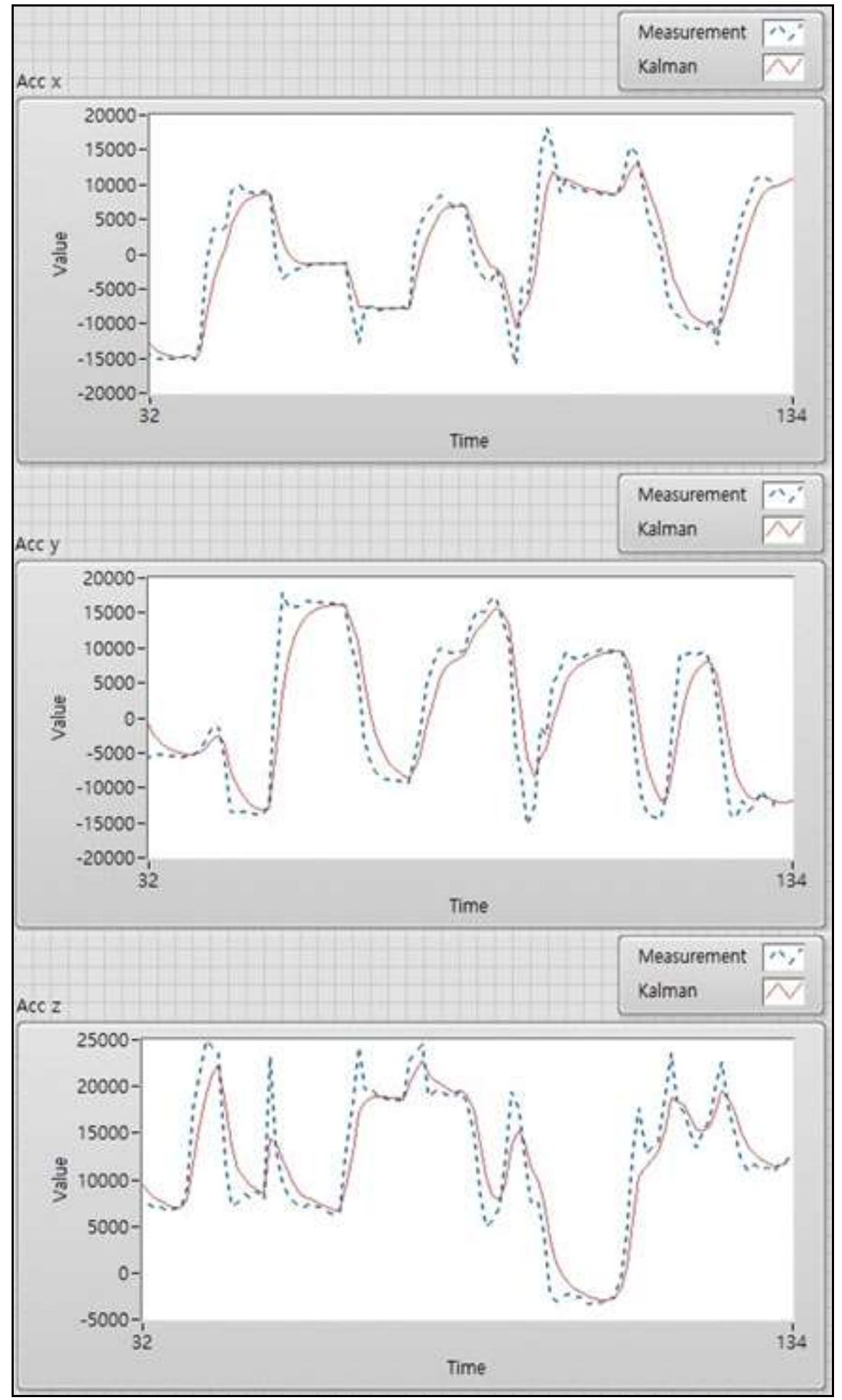

Figure 11. Accelerometer Sensor Graph 


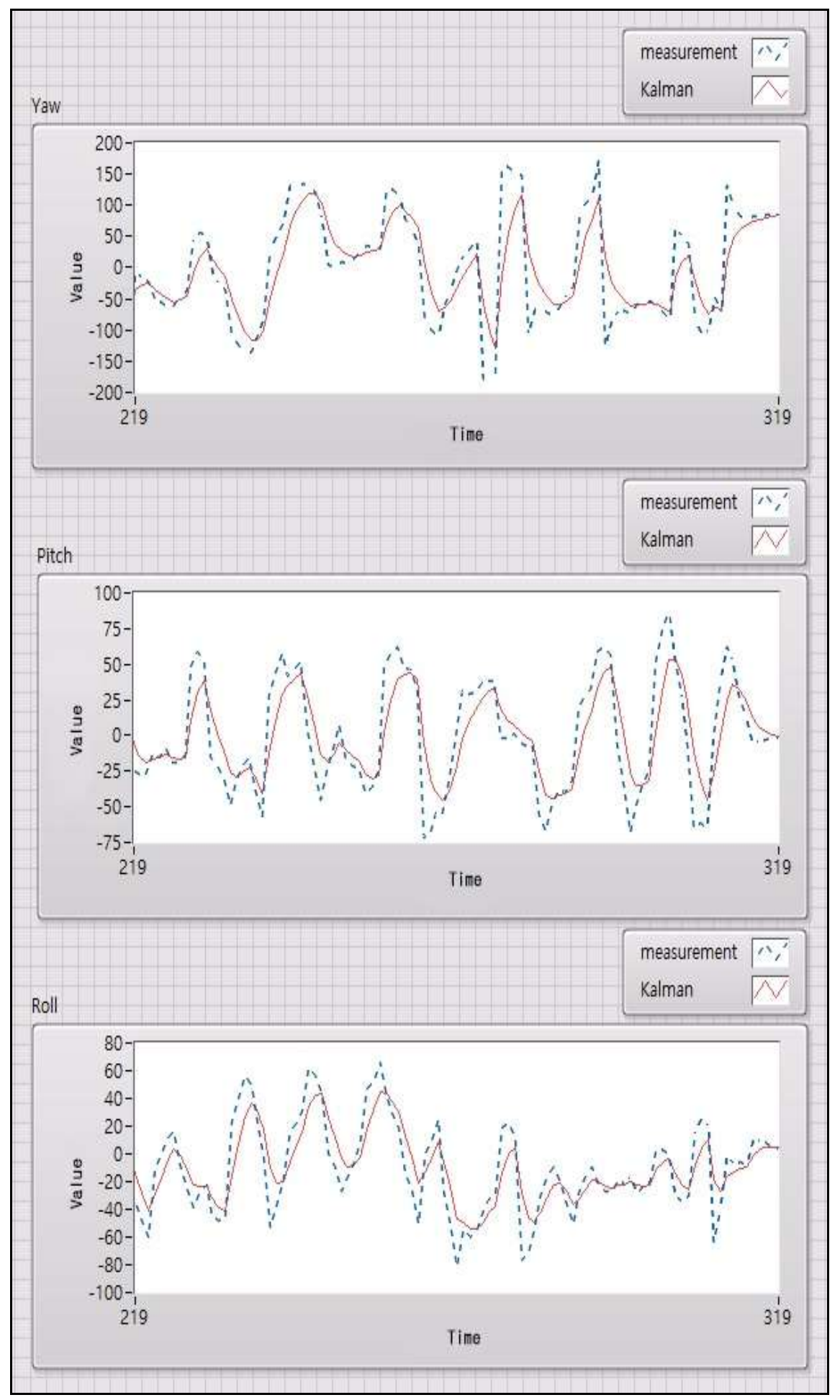

Figure 12. Roll, Pitch, Yaw Graph

In this paper, we evaluate the usability of the developed device by remote experiment. By attaching the motion recognition device to the user's head and collecting data remotely, the data is displayed in LabVIEW graph in real time. Moving the user's head in various directions confirmed the changing data. In addition, we confirmed a more stable data output using the Kalman Filter algorithm [10]. 


\section{Conclusion}

In this paper, we developed a motion recognition device for camera gimbal. By developing a motion recognition device, it can control the object remotely, such as a camera gimbal. That is, the gimbal camera can control the camera in the same way as the head movement of the user. Therefore, this device is more intuitive and convenient recognition. And As shown in Figure 10 Figure 11 Figure 12, the Kalman filter algorithm enables more stable motion. and This device, as well as smart control of the device is applicable to any field that requires the remote control.

We experimented with LabVIEW to test the stability of the developed device. The developed device and the computer were connected wirelessly to confirm the data in real time. And confirmed stable data. The motion recognition check the user's midair motion, and designing the HID-based wireless communication protocol, it is possible to control and easy remote work with the smart device.

\section{References}

[1] K. R. Lee and J. R. Hwang, “A Study on Innovation System with Multi-technology Fusion”, Science and Technology Policy Institute., Korea, (2005).

[2] H.J. Park and T.H. Han, “Augmented Reality-based E-Learning Technology Trends”, Review of Korean Society for Internet Information., vol. 10, no. 2, (2009), pp. 12 22.

[3] X. Liu and J. Li, et al. "A Smart Energy-efficiency Deployment Scheme for Lifetime Enhancement Wireless Sensor Networks", Journal of The Smart Computing Review., vol. 5, no. 6, (2015), pp. 591 601.

[4] I.A. Khan and F. Ahmad, "Application-based Classification and Comparison of Secure Routing Protocols in Wireless Sensor Networks - A Survey", Journal of The Smart Computing Review., vol. 5, no. 3, (2015), pp. 209 223.

[5] D.P. Hong and W.T Woo, "Recent Research Trend of Gesture-based User Interfaces", Telecommunication Review., vol. 18, no. 3, (1981), pp. 403 413.

[6] D. Li and J. Lee, "Minimum Gradient Forwarding for Wireless Sensor Networks", Journal of The Smart Computing Review., vol. 4, no. 5, (2014), pp. 418 425.

[7] L.C. Ko and J. S. Lee, "ZigBee Security for Residential Sensor Networks", Journal of The Smart Computing Review., vol. 1, no. 2, (2011), pp. 95 103.

[8] M.G. Song, at al. "Development of Gimbal System for Aviation Photograph using Smartphone", Korea Society of Precision Engineering. (2014), pp. 294.

[9] A. Mehmood and H. Song, "Smart Energy Efficient Hierarchical Data Gathering Protocols for Wireless Sensor Networks" Journal of The Smart Computing Review., vol. 5, no. 5, (2015), pp. 425 462.

[10] H.O. Jang Editor, "Understanding the LabVIEW Graphics Program", ADC System, Korea, (1998).

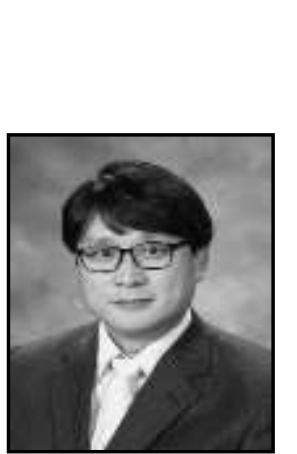

\section{Authors}

Won-Hyuck Choi, graduated from the Ph.d degree in the department of avionics Engineering from Korea Aerospace University in 2006. the Doowon Technical University Smart Network Professor From 2008 to 2014. he is Hanseo University Avionics Engineering Professor in to the present in 2014.

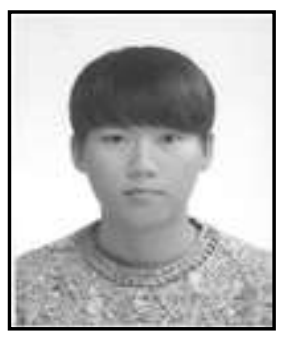

Da-Un Kim, graduate of the Hanseo University avionics bachelor's and a master's degree and Aviation Systems Engineering in 2015. 


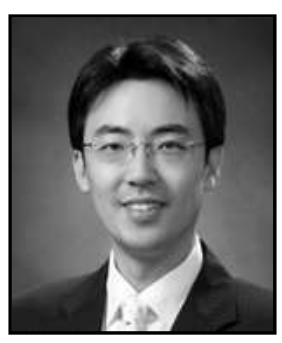

Min-Seok Jie, (Corresponding author), graduated from the Ph.d degree in the department of avionics Engineering from Korea Aerospace University in 2006. he is Hanseo University Avionics Engineering Professor in to the present in 2008. 
International Journal of Control and Automation

Vol. 10, No. 5 (2017) 